\title{
Comparison of the Pain Perception of Conventional and Computer-controlled Anesthesia Techniques for Extraction of Maxillary First Premolar
}

\author{
Varsha Sunil Manekar
}

\begin{abstract}
Introduction: Anterior middle superior alveolar (AMSA) block is a nerve block technique in conjunction with computer-controlled local anesthetic delivery (CCLAD) system. Anterior middle superior alveolar produces local anesthesia (LA) to the maxillary teeth from second premolar to the central incisor including the hard palate and corresponding soft-tissue of both buccal and palatal side, with single palatal injection.
\end{abstract}

Aim: To evaluate efficacy of AMSA block for extraction of maxillary first premolar.

Study design: In randomized single blind, split mouth clinical trial, the 14 patients received the conventional buccal-palatal (B-P) anesthesia on one side and CCLAD anesthesia AMSA for other side. The treatment consisted of bilateral extraction of maxillary first premolars for orthodontics. Pain perception ratings were obtained by using 10 point visual analog scale (VAS).

Results: A total of $100 \%$ cases had painless injection with CCLAD, whereas $28.57 \%$ patients had painless injection experience with conventional LA. In all cases, there was no pain during extraction on both sides. With CCLAD, buccal retraction was painful in four cases whereas palatal retraction was painful in one case.

Conclusion: Anterior middle superior alveolar block injected with CCLAD is better as LA technique for extraction of maxillary first premolar as compared to B-P approach.

Keywords: Anterior superior alveolar nerve block, Computercontrolled local anesthetic delivery system, Greater palatine nerve block, Infraorbital nerve block, Pain perception, Split mouth, Visual analog scale rating.

How to cite this article: Manekar VS. Comparison of the Pain Perception of Conventional and Computer-controlled Anesthesia Techniques for Extraction of Maxillary First Premolar. World J Dent 2015;6(3):150-153.

Source of support: Nil

Conflict of interest: None

\section{INTRODUCTION}

Computer-controlled local anesthetic delivery (CCLAD) has been developed to reduce overall pain perception

Associate Professor

Department of Oral and Maxillofacial Surgery, Government Dental College and Hospital, Nagpur, Maharashtra, India

Corresponding Author: Varsha Sunil Manekar, Associate Professor, Department of Oral and Maxillofacial Surgery, 38, Income Tax Colony, Pratap Nagar, Nagpur-440022, Maharashtra, India Phone: 9823077626, e-mail: varsha@manekar.info related to the local anesthesia (LA) injection. In conjunction with this technology, anterior middle superior alveolar (AMSA) nerve block has been introduced. ${ }^{1}$ The traditionally used LA delivery system is aspirating disposable syringe and needle. A simple mechanical instrument that dates back to 1853, when Charles Pravez patented the first syringe. ${ }^{2}$ The operator controls the penetration of needle, drug infusion by applying pressure on the piston. The local anesthetic is pushed in tissues at variable rate of flow and pressure. There is pain during insertion, LA drug infusion, fear for the needle and syringe. The additional anatomical structures like lip, cheeks, nostrils are anesthetized as per the nerve supply. The first of CCLAD devices, from Milestone Scientific called as 'the Wand' was introduced in 1997. Computer controlled local anesthetic delivery (Fig. 1). It uses the light weight hand piece which is held in pen grasp that provides increased tactile sensation and control compared with the traditional syringe. The rate of flow of local anesthetic solution are controlled by computerized system. As a result there is a consistency of flow of solution in each injection. The flow rate is precise and the pressure is maintained while solution is delivered in tissues. ${ }^{3}$ The Wand is a total new experience in induction of local anesthesia. The CCLAD system is programmed to deliver the local anesthetic drug. The needle position is the only factor under control of operator. According to Hoffmann, Axthelm $\mathrm{W}$, the greater control over the syringe and the fixed flow rates of the LA drug are responsible for a significantly improved injection experience, this is also demonstrated in many clinical studies conducted with CCLAD devices in dentistry. ${ }^{2}$ Anterior middle superior alveolar was first reported by Friedman and Hochman in 1997 during development of CCLAD. ${ }^{4}$ Anterior middle superior alveolar produces LA to the maxillary teeth from second premolar to the central incisor including the hard palate and corresponding soft-tissue of both buccal and palatal side, with single palatal injection.

\section{AIM}

To evaluate efficacy of AMSA block for extraction of maxillary first premolar. 


\section{STUDY DESIGN}

This is a clinical trial of new LA block technique, AMSA for extraction of maxillary first premolar. The study design is randomized, single blind and split mouth.

\section{Inclusion Criteria}

Patients aged 15 to 21 years of both the genders. The bilateral maxillary first premolar erupted in anatomical position, advised for extraction as part of orthodontic correction.

\section{Exclusion Criteria}

Those with any systemic illness including those which contraindicates use of LA, extremely uncooperative patients, patients allergic to local anesthesia and those on sedative medications.

\section{MATERIALS AND METHODS}

- Both right and left maxillary first premolar indicated for extraction by orthodontist were extracted in the same appointment. All injections consisted of $2 \%$ xylocaine with 1:100,000 adrenaline. All procedures were carried out by one operator.

- The procedure along with possible risks and benefits, were explained fully to parents/guardians and subject and their informed consent was obtained. The study was approved by Institutional Ethical Committee.

- The selection of side was randomized by picking of chit by the assistant. The patient was explained about both LA techniques but the use was masked, so the preference of LA and side was blinded for patient.

- The maxillary first premolar is supplied with middle superior alveolar (MSA) nerve; (by anterior superior alveolar (ASA) nerve in case MSA nerve is absent) and on palatal side by terminal branches of greater palatine nerve. On one side the patients received infraorbital nerve block (IONB) and greater palatine nerve block (GPNB) with conventional syringe, and disposable 25 gauge by 1 inch needle. The average amount of solution injected was 1.5 and $0.5 \mathrm{ml}$ respectively. This was followed by extraction of maxillary first premolar.

5. Anterior middle superior alveolar block injected on the other side using the Wand ${ }^{\mathrm{TM}}$ (Milestone Scientific, Inc. Livingston, NJ) (Fig. 1) followed by extraction. Figure 2 shows the injection site of AMSA, on the hard palate about halfway along an imaginary line connecting the mid palatal suture to the free gingival margin, along the line at the contact point between the premolars. Figure 3 shows injection system of CCLAD. In this a conventional local anesthetic cartridge, is

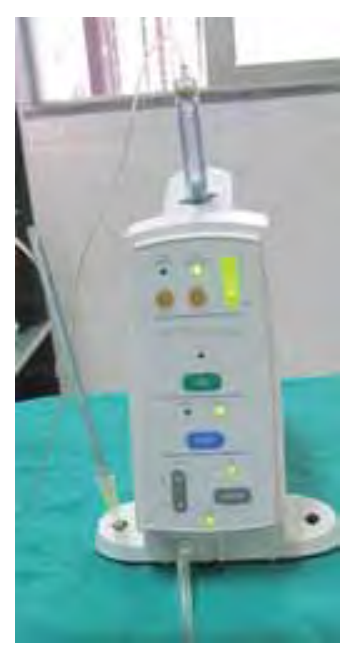

Fig. 1: Computer-controlled local anesthetic delivery system

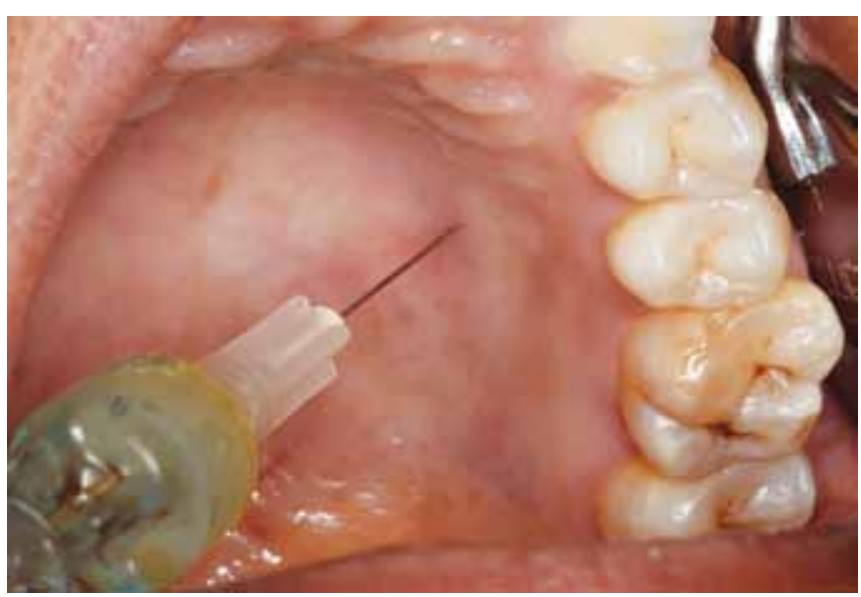

Fig. 2: Injection site of AMSA

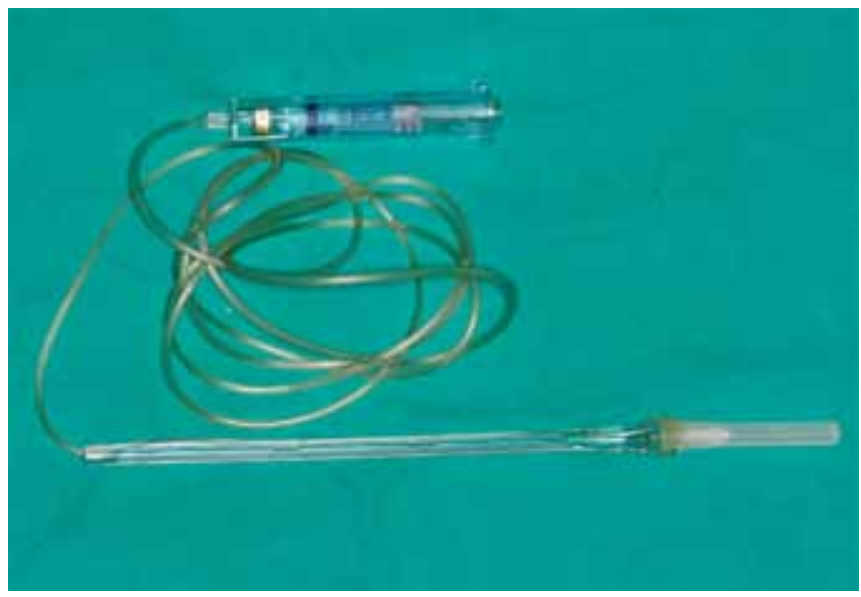

Fig. 3: Wand, injection system of CCLAD

linked by microtubing to a disposable, lightweight, pen like handle, wand with a Luer lock needle (30 gauge) attached. The flow of solution is regulated by foot control. The flow rate is $0.5 \mathrm{ml} / \mathrm{min}$ the precision of pressure and volume ratio is advantage over the variable pressure used in traditional syringe. The average amount of solution injected was $1.4 \mathrm{ml}$.

- Pain perception ratings were obtained by using 10 point visual analog scale (VAS) of pain rating (Fig. 4) 
Pain rating scale ${ }^{\circ}$ Mosby

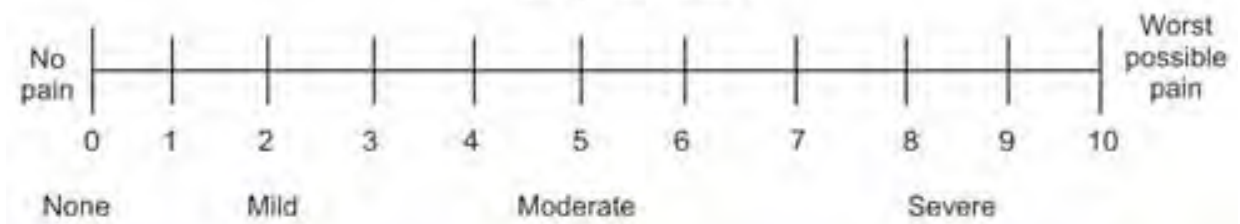

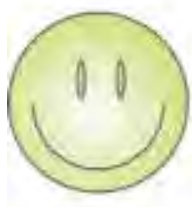

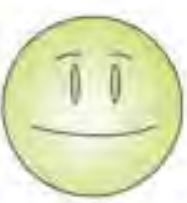

0

No hurt

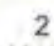

Hurts

little bit

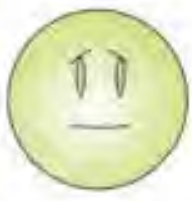

4

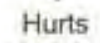

little more

even more

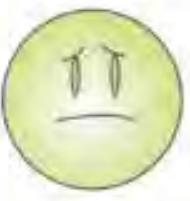

6

Hurts

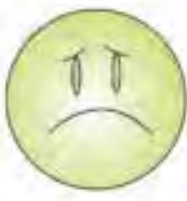

8

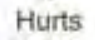

whole lot

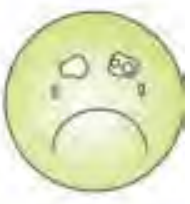

10

Hurts

worst for-pain while injection; on buccal gingival retraction; on palatal gingival retraction, and during extraction.

- Patient's preference for wand or conventional syringe was noted.

\section{OBSERVATIONS}

Total 14 patients participated, received 28 injections followed by extraction of bilateral maxillary first premolar. Mean age group is 17.36 years. There are 12 females and two males patients. For pain perception of nerve block injection on VAS for IONB $28.57 \%$ had ' 0 ' rating, $67.14 \%$ had ' 2 ' rating, $14.28 \%$ with ' 4 ' rating. Similar observations for GPNB, $0 \%$ for ' 0 ' $42.85 \%$ for ' 2 ', $67.14 \%$ for ' 4 '. Anterior middle superior alveolar shows $100 \%$, ' 0 ' rating. Thus pain perception in AMSA injection is painless. Table 1 shows the percentage of VAS rating of pain on injection. The pain rating of extraction of CCLAD anesthesia is shown in Graph 1. Zero score for 10 cases of buccal reflection; ' 2 ' score for 4 cases. Whereas in all 14 cases, the palatal reflection and extraction is with ' 0 ' score. In case of extraction with conventional anesthesia (Graph 2), ' 0 '-no pain for reflection of buccal gingiva and during extraction in all 14 cases. While only in one case the rating is ' 2 ' for palatal reflection, in rest of 13 cases rating is ' 0 '. Thus, the extraction of tooth in both systems is painless. The patient's preference of system shows four patients preferred conventional blocks whereas 10 patients preferred wand system.

\section{RESULTS}

A total of $100 \%$ cases had painless injection with CCLAD, whereas $28.57 \%$ patients had painless injection experience with conventional LA. In all cases, there was no pain during extraction on both sides. With CCLAD, buccal retraction was painful in four cases.
Table 1: The percentage of VAS rating for pain on injection

\begin{tabular}{llll}
\hline & \multicolumn{3}{c}{ Injection pain VAS rating } \\
\cline { 2 - 4 } Nerve block & 0 & 2 & 4 \\
\hline IONB & 28.57 & 67.14 & 14.28 \\
GPNB & 0 & 42.85 & 67.14 \\
AMSA & 100 & 0 & 0 \\
\hline
\end{tabular}

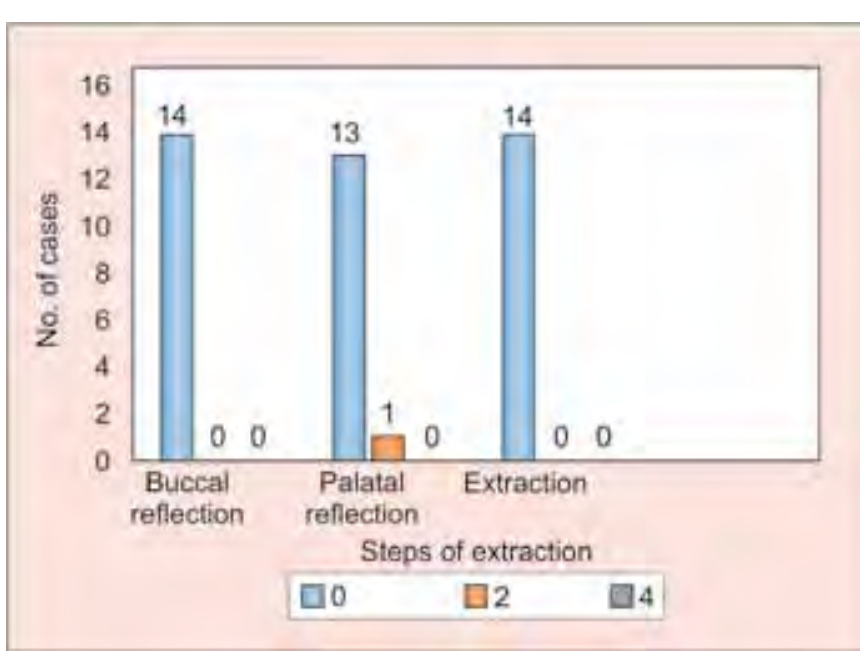

Graph 1: Visual analog scale rating of pain while extraction of first premolar under CCLAD

\section{DISCUSSION}

The AMSA block provides pulpal anesthesia to the maxillary incisors, canines and premolars on the side of injection. Soft-tissue anesthesia is achieved for the entire hard palate on that side and the intraoral mucosa of the five anesthetized teeth, without numbness of the face, lips, and muscles of facial expression. ${ }^{4}$ This technique requires slow administration of $0.5 \mathrm{ml} / \mathrm{min}, 0.9$ to $1.4 \mathrm{ml}$ dosage are recommended. There is reduced pain perception while use of injection technique using wand. This was first demonstrated by Mark Hochman et al. Wand delivers anesthetic at a constant slow rate and controlled pressure, regardless of resistance within tissue. The manufacturer has proposed that the computerized 


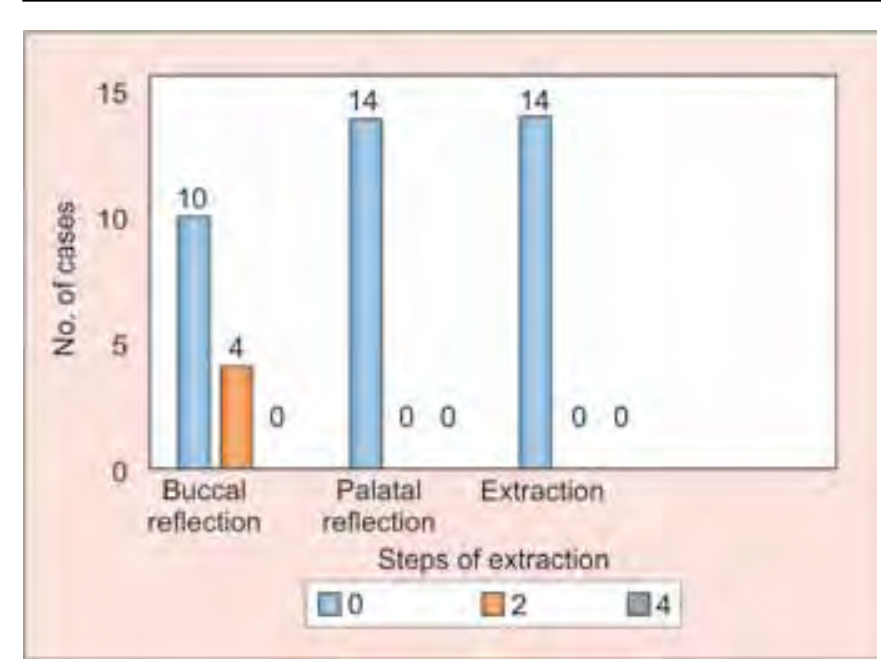

Graph 2: Visual analog scale rating of pain while extraction of first premolar under conventional LA

system delivers anesthetic at a rate below the threshold of pain allowing for a potentially pain free injection. The anesthetic profuse the porous bone of the maxilla and produces profound anesthesia of teeth as well as adequate buccal/ facial anesthesia are achieved with one injection. The adjacent anatomical structures like lip, cheek are not anesthetized reducing patients discomfort. Whereas patients receiving IONB and GPNB with conventional syringe had anesthesia of upper lip, ala of nose, lower eyelid and complete palate on the side of block, soft-tissue anesthesia of buccal and palatal side; pulpal anesthesia to the maxillary incisors, canines and premolars.

In one study carried out by Perry and Loomer the traditional syringe was compared to CCLAD delivery of local anesthetic solution for quadrant scaling and root planning. The AMSA nerve block was received by twenty subjects. Scores for the AMSA injection revealed a highly significant difference in favor of the computer-controlled device $(\mathrm{p}<0.0001){ }^{5}$

In a controlled clinical study with 50 dentists as participants, CCLAD was compared to standard manual syringe for palatal injection, $48(96 \%)$ preferred the CCLAD injections. In comparison pain perception was reduced two- to threefold with CCLAD, in a randomized clinical trial conducted by Nicholson et al. In this study, two operators administered four different types of dental injections, comparing CCLAD to a standard syringe. ${ }^{6,7}$
There are no studies reported to evaluate the efficacy of AMSA, a single injection LA block technique for extraction of maxillary first premolar, compared to traditional B-P, LA blocks. This clinical trial shows obvious advantages of AMSA are profound anesthesia of teeth (second premolar to central incisor of same side), single pain free injection, minimum amount of solution, no numbness of lips, cheek and nostril. The sample size of this study is small. The extraction in both systems is painless, but CCLAD with its AMSA has obvious advantages over conventional B-P LA as mentioned above.

\section{CONCLUSION}

The new block AMSA block is better for local anesthesia of maxillary first premolar as compared to conventional buccal-palatal approach. Occasionally, the buccal anesthesia may not be effective (4 out of 14 cases in this case). Thus, injection with CCLAD is painless and effective for extraction. Further research with bigger sample size is suggested.

\section{REFERENCES}

1. Friedman MJ, Hochman MN. Using AMSA and P-ASA nerve blocks for esthetic restorative dentistry. Gen Dent 2001;49(5):506-511.

2. Hoffmann, Axthelm W. History of dentistry, Chicago, Quintessence 1981. p. 339.

3. Stanlay F Malamed. Handbook of local anesthesia, Mosby. 5th ed. 2004;90-94.

4. Friedman MJ, Hochman MN. The AMSA injection: a new concept for local anesthesia of maxillary teeth using a computer-controlled injection system. Quintess Int 1998;29(5): 297-303.

5. Perry DA, Loomer PM. Maximizing pain control. The AMSA injection can provide anesthesia with few injections and less pain. Dimensions of Dent Hygiene 2003;1(2):28-33.

6. Nicholson JW, Berry TG, Summitt JB, Yuan CH, Witten TM. Pain perception and utility: a comparison of the syringe and computerized local injection techniques. General Dentist 2001;4(2):167-172.

7. Chaudhry K, Shishodia M, Singh C, Tuli A. Comparative evaluation of pain perception by vibrating needle (Vibraject ${ }^{\mathrm{TM}}$ ) and conventional syringe anesthesia during various dental procedures in pediatric patients: a short study. Int Dent Med J Adv Res 2015;1:1-5. 